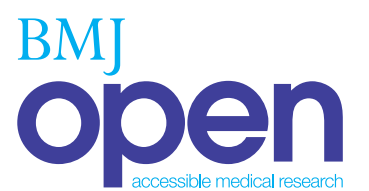

\title{
Identifying clinical criteria to predict Type 1 diabetes, as defined by absolute insulin deficiency: a systematic review protocol
}

\author{
Beverley M Shields, ${ }^{1}$ Jaime L Peters, ${ }^{2,3}$ Chris Cooper, ${ }^{2}$ Roy J Powell, ${ }^{4}$ \\ Bridget A Knight, ${ }^{1}$ Christopher Hyde, ${ }^{2}$ Andrew T Hattersley ${ }^{1}$
}

To cite: Shields BM, Peters JL, Cooper C, et al. Identifying clinical criteria to predict Type 1 diabetes, as defined by absolute insulin deficiency: a systematic review protocol. BMJ Open 2012;2: 002309 .

doi:10.1136/bmjopen-2012002309

- Prepublication history and additional material for this paper are available online. To view these files please visit the journal online (http://dx.doi.org/10.1136/ bmjopen-2012-002309).

Received 6 November 2012 Revised 6 November 2012 Accepted 20 November 2012

This final article is available for use under the terms of the Creative Commons Attribution Non-Commercial 2.0 Licence; see http://bmjopen.bmj.com

For numbered affiliations see end of article

Correspondence to Dr Beverley Shields, Beverley.Shields@pms.ac.uk

\section{ABSTRACT}

Introduction: Management of a patient's diabetes is entirely dependent upon the type of diabetes they are deemed to have. Patients with Type 1 diabetes are insulin deficient so require multiple daily insulin injections, whereas patients with Type 2 diabetes still have some endogenous insulin production so insulin treatment is only required when diet and tablets do not establish good glycaemic control. Despite the importance of a correct diagnosis, classification of diabetes is based on aetiology and relies on clinical judgement. There are no clinical guidelines on how to determine whether a patient has Type 1 or Type 2 diabetes. We aim to systematically review the literature to derive evidence-based clinical criteria for the classification of the major subtypes of diabetes.

Methods and analysis: We will perform a systematic review of diagnostic accuracy studies to establish clinical criteria that predict the subsequent development of absolute insulin deficiency seen in Type 1 diabetes.

Insulin deficiency will be determined by reference standard C-peptide concentrations. Synthesis of criteria identified will be undertaken using hierarchical summary receiver operating characteristic curves.

Ethics and dissemination: As this is a systematic review, there will be no ethical issues. We will disseminate results by writing up the final systematic review and synthesis for publication in a peer-reviewed journal and will present at national and international diabetes-related meetings.

\section{INTRODUCTION}

Correct classification of a patient's diabetes is crucial to ensure they are provided with the most appropriate treatment, management and education. There are two main subtypes of diabetes: Type 1 diabetes $(\sim 15 \%)$ and Type 2 diabetes $(\sim 83 \%),{ }^{1}$ in addition to other rare genetic or secondary subtypes.

\section{ARTICLE SUMMARY}

Article focus

- Current guidelines for classifying Type 1 and Type 2 diabetes according to WHO, ADA and NICE, are based on aetiology rather than clinical criteria.

- Differences in the treatment and management of Type 1 and Type 2 diabetes are all based on whether a patient is producing their own insulin (Type 1) or not (Type 2).

- We aim to systematically review the literature to identify clinical criteria that can be used to predict absolute insulin deficiency (as seen in Type 1 diabetes) that can be used in the classification of diabetes.

\section{Key messages}

- Misclassification of diabetes has been highlighted as a problem. There are currently no evidence-based criteria that can be used to classify Type 1 and Type 2 diabetes.

- We aim to systematically review the literature to identify clinical criteria that can be used to predict absolute insulin deficiency (as seen in Type 1 diabetes).

- We aim to synthesise the results to produce criteria that can be used in the classification of diabetes.

Strengths and limitations of this study

- Our review will be systematic and exhaustive. Our search strategy encompasses a large number of electronic databases and websites, including those to examine grey literature, and we are not restricting our search to English language only.

- The only clinical criteria currently proposed for classification (from the Royal College of GPS/ NHS Diabetes) are based on expert-opinion-our study will provide evidence-based criteria.

- A validation study will be necessary to determine the utility of the criteria identified in practice. 
Differences in the NICE (National Institute of Clinical Excellnce) guidelines for Type $1^{2}$ and Type $2^{3}$ diabetes are marked and include the treatment used (patients with Type 1 diabetes will start on multiple insulin injections while those with Type 2 diabetes will only go to insulin if diet and tablets do not control blood glucose), the dietary advice given, and the monitoring required by patients and their healthcare team. These major differences are vital to ensure optimal glycaemic control for the patient and hence reduce their long-term risk of complications. The clear differences in clinical care in these patients are entirely based on assumptions of differences in endogenous insulin secretion.

Despite clinical guidance being specific for Type 1 and Type 2 diabetes, there are no published, evidencebased, clinical criteria that can be used to define a patient's type of diabetes. Guidance on the classification of the two types of diabetes from major health organisations is limited, and primarily focuses on aetiology. The WHO defines Type 1 diabetes as ' $\beta$-cell destruction, usually leading to absolute insulin deficiency' mainly occurring as the result of an autoimmune process. ${ }^{4} 5$ Type 2 diabetes is described by $\mathrm{WHO}$ as 'ranging from predominantly insulin resistance with relative insulin deficiency to predominantly an insulin secretory defect with insulin resistance'. 45 Essentially, the key difference between the two types of diabetes is whether the patient continues to produce their own insulin or not. Insulin deficiency/production can be assessed by measurement of C-peptide concentrations in either blood or urine, ${ }^{67}$ but this measurement is rarely carried out in practice. Classification is based on clinical judgement.

Classification used to be relatively easy with Type 1 diabetes being traditionally thought of as a young-onset disease that presents acutely and Type 2 diabetes being associated with obesity and older age. However, these indications are not always helpful. Patients with Type 2 diabetes can present with ketoacidosis and the onset of autoimmune diabetes can be slower in older patients. With the prevalence of obesity increasing in the population as a whole, Type 2 diabetes is being seen in younger patients and Type 1 patients can also be obese, so the distinction between the two types is becoming blurred. Discriminating Type 1 from Type 2 diabetes, particularly in young-onset/middle-aged patients, has become more challenging. ${ }^{8}$ Recent work has shown misclassification of diabetes can occur in up to $13-16 \%$ of $\operatorname{cases}^{9}{ }^{10}$ so the current practice based on aetiological guidelines and clinical opinion is clearly insufficient. A recent report by the working group between NHS Diabetes and The Royal College of General Practitioners has highlighted this problem. ${ }^{10}$ Pragmatic guidelines on diabetes classification have been developed by this group, but are taken from consensus expert clinical opinion rather than being evidence based. ${ }^{10}$

Clearer, evidence-based guidelines on the classification of diabetes are needed to ensure patients are appropriately managed and help reduce the errors in misdiagnosis. As measures of endogenous insulin secretion are rarely carried out in practice, the identification of clinical criteria that could be used as a surrogate to help discriminate the two types of diabetes would be invaluable, and could help form clearer guidelines for clinicians.

We aim to derive evidence-based clinical criteria for the classification of the major subtypes of diabetes. These are needed to inform local and national guidelines so healthcare practitioners can select the appropriate treatment regimes for their patients. The main objective will be to systematically review and synthesise the medical literature to establish clinical criteria that predict the subsequent development of absolute insulin deficiency (measured by $\mathrm{C}$ peptide) seen in Type 1 diabetes.

\section{METHODS AND ANALYSIS \\ Overview}

A systematic review will be undertaken to identify, critique and synthesise the evidence on clinical criteria that predict absolute insulin deficiency that can be used in the classification of Type 1 diabetes. This can be considered a systematic review of diagnostic test accuracy, with insulin deficiency representing the outcome and clinical characteristics representing the predictors. The proposed systematic review will be undertaken following the principles of systematic reviews as set out in NHS Centre for Reviews and Dissemination guidelines. ${ }^{11}$ The protocol has been registered with PROSPERO (http://www.crd. york.ac.uk/prospero/, reference CRD42012001736). The study designs that we will identify as relevant to this systematic review will be diagnostic studies where clinical features, such as age at diagnosis and body mass index (BMI), are compared against insulin deficiency as defined by reference standard C-peptide concentrations.

\section{Population}

Patients diagnosed with diabetes with evidence of insulin deficiency, as determined by negative C-peptide results ('Type 1 diabetes'). Type 2 diabetes will be the exclusion of Type 1 and other subtypes where the aetiology is known (eg, secondary diabetes or monogenic diabetes). The focus of the literature search will be on patients with the more common forms of diabetes (ie, known rare genetic, syndromic or secondary forms of diabetes will be excluded). All ages and racial groups will be considered.

\section{Reference standard}

For 'Type 1 diabetes', reference standard will be measurement of $\mathrm{C}$ peptide to determine insulin deficiency. All measurements will be considered including different types of sample (urine or blood), different types of stimulus (fasting, random, glucose-stimulated, mixed meal-stimulated, postglucagon-stimulated), and timing after stimulation (peak or area-under-curve of numerous 
measurements, $90 \mathrm{~min}, 2 \mathrm{~h}$ ). The definition of insulin deficiency used will be recorded and evaluated.

\section{Comparator/predictor}

Preliminary scoping has indicated that the major predictors will be age at diagnosis and BMI, so the primary focus will be on assessing their diagnostic accuracy and the optimal thresholds of these for discriminating the two types of diabetes. Any clinical features that can be measured and replicated will be considered. Some criteria will be subject to clinician judgement (eg, time to insulin treatment, which is confounded by the clinician considering their patient has Type 1 diabetes). A distinction will be made between those that can be used for classification at diagnosis and those that can be used after diagnosis (eg, time to insulin treatment cannot be used at diagnosis).

\section{Outcomes}

(i) The clinical features assessed to predict C-peptide levels; (ii) the sensitivity and specificity of clinical features as measured against insulin deficiency as determined by $\mathrm{C}$ peptide.

\section{Study design}

Diagnostic accuracy studies.

\section{Search strategy}

Our search approach will be systematic and exhaustive. We propose searching for relevant literature in a variety of ways so as to ensure sensitivity and depth of retrieval. We intend to search not only for peer-reviewed publications but also grey literature. Our search strategy will include

1. Electronic databases (see online supplementary material for details of the databases that will be used in the search strategy)

2. Websites-we propose searching websites as tools for gathering grey-or difficult to locate-literature (see online supplementary material). Given the clinical nature of our topic, it is unlikely these searches will yield much information, so the list is somewhat shorter than for a public health topic.

3. Pearl growing, Snowballing and Hand-searching-in addition to searching electronic databases, we propose citation chasing and contacting the authors of included studies. These approaches have been shown to yield additional information. ${ }^{12} 13$ Forward citation chasing will be conducted in Web of Science (ISI), and bibliographies of included studies will be pursued by the review team to further enhance our ability to capture all relevant literature. We will handsearch the core journals identified by the number of includes in our search, most likely to be Diabetes Care, Diabetologia and Diabetic Medicine as the key clinical diabetes journals.

\section{Search limits}

Searches will be limited to the date range 1979-present, which reflects the original classification scheme proposed by the National Diabetes Data Group. ${ }^{14}$ The searches will also be limited to human only populations. We will not be restricting the search by language. ${ }^{15}$

\section{Search recording}

The exported files from the literature searching will be uploaded and de-duplicated in Endnote X4 (Thomson Reuters) and then imported into Microsoft Access for reviewing purposes. Where an export is not possible, for example, from a resource without RIS functionality, the data will be exported to a word file and manually entered into Endnote and Access. All files will be copied and saved for record. The searches will be recorded using PRISMA guidelines. ${ }^{16}$ An example of this search recoding can be seen in online supplementary material.

\section{Update searches}

Towards the end of the review time line, we will repeat the search process to bring our review up to date.

\section{Study selection}

There will be two stages to the identification of relevant studies to be included in the systematic review. First, the titles and abstracts of all references identified by the above search strategy will all be double-screened by two reviewers. Any disagreements on inclusion of studies between the two reviewers will be resolved by discussion, or the inclusion of a third reviewer if necessary. Second, the full text of all references identified as included at the first stage will be obtained and then assessed by both reviewers. The references of all relevant studies will be searched for any further relevant studies.

\section{Data extraction}

A standard data extraction form will be developed and applied to all included studies. This data extraction form will be piloted at the beginning of the research to ensure it is sufficient and captures all relevant information. The information extracted from each study will include: setting (eg, country, year), general patient demographics (eg, age range, gender, racial group, type of diabetes), measurement of insulin deficiency (eg, test threshold, sample type), results (eg, predictors of $\mathrm{C}$ peptide, magnitude of effect, statistical significance, sensitivity and specificity of predictor) and conclusions.

\section{Study quality assessment}

The QUADAS-2 (Quality Assessment of Diagnostic Accuracy Studies) tool ${ }^{17}$ will be used to assess the quality of the diagnostic accuracy studies included in the systematic review. This tool will help identify potential biases in the primary studies so that the conclusions of the systematic review appropriately reflect the possible impact of such biases. Particular attention will be paid to the reference standard, where details of the sample type, sample 
collection, stimulus used, storage, assay and cut-off used will be recorded and assessed. Timing of measurements in relation to diagnosis will also be recorded.

\section{Data synthesis and exploration of bias}

Data analysis will comprise four steps. The first will be the initial visualisation of the data by plotting the sensitivity and specificity from all the included studies on the ROC (receiver operating characteristic) space. This will allow some assessment of the heterogeneity of the estimates across the studies.

Second, we will use the Hierarchical Summary ROC model $^{1819}$ to determine the overall diagnostic value and sensitivity and specificity of individual diagnostic characteristics at different thresholds. ${ }^{20}$ It is envisaged that age at diagnosis and BMI will be the two main predictors for absolute insulin deficiency. Univariate analysis of the diagnostic value of these predictors (and any other clinical features identified) will initially be conducted, and, in cases where papers have reported two criteria together linked ROC plots ${ }^{20}$ can be used to display how the variables perform in combination. Where such analysis cannot be conducted, combinations will be considered on a rational basis. The reference standard is likely to differ across the studies included in the systematic review; therefore, subgroup analysis may need to be carried out to explore the impact of the potential bias in this. Hierarchical summary ROC models also incorporate random study effects enabling heterogeneity between studies to be accounted for, but not explained.

The third step will be to investigate possible sources of heterogeneity by the addition of covariates to the hierarchical summary ROC models. As different criteria may apply to different groups, these covariates will include age (adults vs children), race and gender. However, such analyses are limited by the number of studies included in the systematic review and should be considered exploratory as they are based on the study level covariates. ${ }^{21}$ It will be important to separate out the criteria which can be used at diagnosis and the criteria which can be used after diagnosis.

Finally, we will investigate evidence on the existence of reporting or publication bias within the systematic review. Investigations of possible reporting or publication bias in any systematic review are impacted by there being a small number of included studies and between-study heterogeneity. Therefore, exploratory analysis using funnel plots and, if appropriate, a related test for funnel plot asymmetry ${ }^{22}$ will be undertaken.

\section{Initial scoping}

The search syntax we have tested in our preliminary scoping searches has been included in online supplementary material. Applying this syntax to the two primary databases, Medline and Embase, yields 5804 and 8566 results, respectively. Given that these two searches will include considerable duplication (due to the crossover between these two databases) and that further studies will be identified from further databases, we are estimating we will end up with 10000 results for screening. Approximately $10 \%$ of all search results in our Medline scope were non-English language, however, titles and abstracts are often available in English. From initial scoping we are estimating we will identify 200 abstracts from which we will need to obtain full text, of which we expect that, at the most, two will be of non-English language and will require translation. From the final review of these articles, we anticipate 20 will be suitable for the final synthesis.

\section{Ethics and dissemination}

As this is a systematic review there will be no ethical issues. We will disseminate results by writing up the final systematic review and synthesis for publication in a peerreviewed journal (reported using preferred reporting items for systematic reviews and meta-analyses (PRISMA) guidelines) and will present at the national and international diabetes-related meetings.

\section{DISCUSSION}

A systematic review of the literature on predictors of insulin deficiency is an important first step in determining evidence-based guidelines that can be used for classification of diabetes. By examining the current evidence base, we can synthesise information on existing predictors to determine a useful set of clinical criteria. The next step will be to validate these criteria against gold standards of insulin deficiency in datasets of patients with $\mathrm{C}$ peptide measured.

\section{Author affiliations}

${ }^{1}$ NIHR Exeter Clinical Research Facility, University of Exeter Medical School, University of Exeter, Exeter, UK

${ }^{2}$ Peninsula Technology Assessment Group, University of Exeter Medical School, University of Exeter, Exeter, UK

${ }^{3}$ Peninsula Collaborations for Leadership in Applied Health Research and Care, University of Exeter Medical School, University of Exeter, Exeter, UK

${ }^{4}$ Research Design Service South West, Royal Devon and Exeter NHS Foundation Trust, Exeter, UK

Contributors BS and JP designed the protocol and drafted the manuscript. $\mathrm{AH}$ conceived the original idea and was involved in the design of the study and approved the final manuscript. CC devised the search strategy and critically revised drafts of the manuscript. $\mathrm{RP}, \mathrm{BK}$ and $\mathrm{CH}$ advised on the study design and critically revised drafts of the manuscript. All authors approved the final manuscript.

Funding This study was funded by the National Institute for Health Research (NIHR) under its Research for Patient Benefit (RfPB) programme (PB-PG-0711-25111). The views expressed are those of the author(s) and not necessarily those of the NHS, the NIHR, or the Department of Health, UK.

Provenance and peer review Not commissioned; internally peer reviewed.

Data sharing statement No additional data are available.

\section{REFERENCES}

1. Diabetes UK. Diabetes in the UK 2010 (Mar 2010): Key statistics on diabetes, 2010.

2. National Institute for Clinical Excellence. Clinical Guideline 15: Type 1 diabetes: diagnosis and management of type 1 diabetes in children, young people and adults. London: NICE, 2010. 
3. The National Collaborating Centre for Chronic Conditions. TYPE 2 DIABETES: National clinical guideline for management in primary and secondary care (update). London: NICE, 2011.

4. International Diabetes Federation. Definition and diagnosis of diabetes mellitus and intermediate hyperglycaemia: report of a WHO/IDF consultation. Geneva: WHO, 2006.

5. American Diabetes Association. Diagnosis and classification of diabetes mellitus. Diabetes Care 2010;33(Suppl 1):S62-9.

6. Clark PM. Assays for insulin, proinsulin(s) and C-peptide. Ann Clin Biochem 1999;36(Pt 5):541-64.

7. Polonsky K, Frank B, Pugh $\mathrm{W}$, et al. The limitations to and valid use of C-peptide as a marker of the secretion of insulin. Diabetes 1986;35:379-86.

8. Farmer A, Fox R. Diagnosis, classification, and treatment of diabetes. BMJ 2011;342:d3319.

9. de Lusignan S, Khunti K, Belsey J, et al. A method of identifying and correcting miscoding, misclassification and misdiagnosis in diabetes: a pilot and validation study of routinely collected data. Diabet Med 2010;27:203-9.

10. NHS Diabetes and Royal College of General Practitioners Working Group. Coding, Classification and Diagnosis of Diabetes. Secondary Coding, Classification and Diagnosis of Diabetes. 2010. http://www. diabetes.nhs.uk/areas_of_care/diagnosis_and_ongoing_care/ classification_of_diabetes/ (accessed 17 Dec 2012)

11. CRD. Systematic reviews: CRD's guidance for undertaking reviews in healthcare. York: Centre for Reviews and Dissemination, 2009.

12. Greenhalgh T, Peacock R. Effectiveness and efficiency of search methods in systematic reviews of complex evidence: audit of primary sources. BMJ 2005;331:1064-5.

13. Papaioannou $D$, Sutton $A$, Carroll $C$, et al. Literature searching for social science systematic reviews: consideration of a range of search techniques. Health Info Libr J 2010;27:114-22.
14. Classification and diagnosis of diabetes mellitus and other categories of glucose intolerance. National Diabetes Data Group. Diabetes 1979;28:1039-57.

15. Moher D, Pham B, Klassen TP, et al. What contributions do languages other than English make on the results of meta-analyses? J Clin Epidemiol 2000;53:964-72.

16. Liberati A, Altman DG, Tetzlaff $\mathrm{J}$, et al. The PRISMA statement for reporting systematic reviews and meta-analyses of studies that evaluate health care interventions: explanation and elaboration. PLOS Med 2009;6:e1000100-e00.

17. Whiting PF, Rutjes AWS, Westwood ME, et al. QUADAS-2: a revised tool for the quality assessment of diagnostic accuracy studies. Ann Intern Med 2011;155:529-36.

18. Rutter CM, Gatsonis CA. A hierarchical regression approach to meta-analysis of diagnostic test accuracy evaluations. Stat Med 2001;20:2865-84.

19. Rutter CM, Gatsonis CA. Regression methods for meta-analysis of diagnostic test data. Acad Radiol 1995;2 Suppl 1:S48-56; discussion S65-7, S70-1 pas.

20. Macaskill P, Gatsonis C, Deeks JJ, et al. Analysing and presenting results. In: Deeks JJ, Bossuyt PM, CG, eds. Handbook for systematic reviews of diagnostic test accuracy version 1.0. The Cochrane Collaboration, 2010. http://srdta.cochrane.org/sites/srdta. cochrane.org/files/uploads/Chapter\%2010\%20-\%20Version\%201.0. pdf (accessed 17 Dec 2012)

21. Higgins J, Green S. Cochrane handbook for systematic reviews of interventions VERSION 5.1.0 (updated March 2011). The Cochrane Collaboration, 2011. http://www.cochrane-handbook.org (accessed 17 Dec 2012)

22. Deeks JJ, Macaskill P, Irwig L. The performance of tests of publication bias and other sample size effects in systematic reviews of diagnostic test accuracy was assessed. J Clin Epidemiol 2005;58:882-93. 